\title{
ICE WATER CLASSIFICATION USING STATISTICAL DISTRIBUTION BASED CONDITIONAL RANDOM FIELDS IN RADARSAT-2 DUAL POLARIZATION IMAGERY
}

\author{
Y. Zhang a, c, F. Li ${ }^{\text {a,b,c* }}$, S. Zhang ${ }^{\text {a,c }}$, W. Hao ${ }^{\text {acc }}$, T. Zhu ${ }^{\text {b }}$, L. Yuan ${ }^{\text {a,c }}$, F. Xiao ${ }^{\text {a,c }}$ \\ ${ }^{a}$ Chinese Antarctic Center of Surveying and Mapping, Wuhan University, 430079 Wuhan, China (yuzhang_spl@whu.edu.cn, \\ fli@whu.edu.cn, zskai@whu.edu.cn, hwf_369@126.com, shaw89@whu.edu.cn) \\ $\mathrm{b}$ The State Key Laboratory for Information Engineering in Surveying, Mapping and Remote Sensing, Wuhan University, 430079 \\ Wuhan, China - zhutingting62008@163.com \\ ${ }^{\mathrm{c}}$ Collaborative Innovation Centre for Territorial Sovereignty and Maritime Rights, Wuhan University, 430079 Wuhan, China
}

Commission WG III/9

KEY WORDS: Sea Ice Classification, SAR, Conditional Random Fields, Statistical Distribution Model

\begin{abstract}
:
In this paper, Statistical Distribution based Conditional Random Fields (STA-CRF) algorithm is exploited for improving marginal ice-water classification. Pixel level ice concentration is presented as the comparison of methods based on CRF. Furthermore, in order to explore the effective statistical distribution model to be integrated into STA-CRF, five statistical distribution models are investigated. The STA-CRF methods are tested on 2 scenes around Prydz Bay and Adélie Depression, where contain a variety of ice types during melt season. Experimental results indicate that the proposed method can resolve sea ice edge well in Marginal Ice Zone (MIZ) and show a robust distinction of ice and water.
\end{abstract}

\section{INTRODUCTION}

In the marine cryosphere, sea ice dominates the exchanges between ocean and atmosphere, by reflecting solar radiation, and impacts on ecosystem. Sea ice plays as a positive indicator in global climate change system. Due to the limitation of harsh weather conditions in polar regions, remote sensing has become a potential technique for monitoring daily sea ice, especially the microwave remote sensing has been widely used in retrieving sea ice concentration and extent to characterize the spatial and temporal sea ice evolution. From the continuous observations of remote sensors including SSM/I, AMSR-E and AMSR-2, sea ice extent over the Antarctic and Arctic show contrasting trends. However, high uncertainty of sea ice within thin ice zone results from coarse (kilometer-scale) spatial resolutions of passive microwave remote sensing data (Shokr et al., 2012). During the melt season, the deformation features caused by the wind and ocean forcings including the rafting and ridging is challenging for ice water discrimination from remote sensing data (Heygster et al., 2012). Synthetic Aperture Radar (SAR) can provide rich details for describing sea ice types. Studies of sea ice signatures in C-band SAR images over the last two decades have shown that a number of ice parameters can be determined from the images such as ice edge; ice typesmultiyear, first-year, young, and new ice; fast ice boundaries; ice drift and shear zones; areas of level and deformed ice; leads; polynyas; and some other parameters. This implies that quantitative information about forms of ice, stage of development, and concentration should be derived from SAR images. However, the marginal edge of each sea ice types is still ambiguous limited by the algorithm. In this paper, a novel method is developed by integrating the statistical distribution potential into a conditional random field framework.

Sea-ice segmentation of SAR imagery is a very difficult task due to the presence of a multiplicative noise known as speckle noise. Not only does speckle noise degrade the quality of SAR images but it also makes it a very challenging task to extract tonal and texture information from SAR. Few methods have been proposed for SAR sea ice classification. The previous works in Karvonen and David Clausi (Clausi et al., 2010) have illustrated some robust methods without evaluation. However, operational approach is urged for sea ice classification from the high spatial resolution SAR images.

SVM treats each pixel individually without considering the spatial information in sea ice analysis (Li et al., 2015). CNNSFCRF takes the dual polarization SAR data for sea ice concentration by considering the contextual information. Firstly, $\mathrm{CNN}$ is used for initial sea ice concentration and then SFCRF has been used for refining the initial sea ice concentration. However, CRFs can be directly utilized for ice water classification in this paper. CRFs model the conditional distribution over labels field given the observations field (Zhu et al., 2016). In this paper, CRFs have been proposed for ice water classification considering the statistical information of SAR backscatter characteristics.

In SAR image, the basic quantity measured by SAR at each pixel, with some provisos, is fundamentally determined by electromagnetic scattering processes (Olive et al., 1998), and it can be represented by the number of discrete scatters in each resolution cell. With the Gaussian assumption, the amplitude shown to be Rayleigh distributed, while the intensity has a negative exponential pdf. These two models are valid only for the single-look SAR images with homogeneous areas, but the negative exponential distribution can be further extended to the Nakagami-Gamma model for the cases of multi-look SAR images (Goodman et al., 1975). In many cases of practical interest, the non-Gaussian behaviours are observed from actual SAR measurements; hence, the above Gaussian conjecture should not generally be confirmed. Then, the symmetric alphastable distribution (Kuruoglu et al., 2004) and the generalized Gaussian probability density function (pdf) (Moser et al., 2006) are proposed, thus resulting in the heavy-tailed Rayleigh and 
generalized Gaussian Rayleigh (GGR) models of amplitude SAR images, respectively. Recently, another distribution, namely generalized Gamma Rayleigh ( $\mathrm{Li}$ et al., 2010), is derived in the same way with the help of two-sided generalized Gamma distribution. For the parameter estimation of probability density functions, the most frequently employed classical methods of statistical parameter estimation are the maximum likelihood (ML), the method of moments (MoM) (Schervish 2012) approaches and Method of Logarithmic Cumulants (MoLC). The theoretical statistical properties of ML are well established under several regular conditions. However, the PDF models involve complicated analytical expressions and do not originate from the exponential family of distributions, ML could not guarantee the attractive asymptotical properties. For MoM, it outperforms by ML in the exponential family of distributions including gamma and Gaussian PDF. However, the applicability of this method is restricted by the existence of finite moments up to the necessary order, which is not the case for several critical scenarios. Moreover, on the basis of high order statistics, MoM is sensitive to outliers that are inherent in real signals due to noise or registration errors (Li et al., 2016). MoLC is a parameter estimation technique developed specifically for positive-valued PDF. Most SAR-specific statistical models account for speckle, and therefore constitute multiplicative models, which renders them well-suited for the Mellin transform and MoLC (Oliver et al., 2004).

In order to design a robust SAR ice water analysis algorithm, system statistical distribution models have been exploited for discriminating ice and water in this paper. The clear ice water boundaries can be obtained by incorporating the statistical information and spatial information into STA-CRF framework for improving ice water discrimination. To calculate statistically significant estimates using five statistical distribution models is useful for ice water analysis. With the SAR data increasing, robust algorithm should be developed for sea ice analysis in high spatial resolution SAR imagery with less time consumption.

The goal of this study is to propose a robust statistical distribution for describing the sea ice characteristics under CRF framework, which is important for deriving the spatial contextual distribution. The statistical distribution parameters aim at describing the scattering characteristics for different ice types and open water, since the statistical distribution model reveals the statistical characteristics of electromagnet in SAR imaging. It serves as a feature descriptor in the CRF model. Then the graph cut algorithm is exploited to obtain sea ice classification results. This paper is organized as follows. Section 2 describes the statistical distribution based CRF sea ice classification method combining statistical distribution modeling. Then, the methodology description including statistical distribution parameter estimation and the CRF framework is given in Section 3. Section 4 presents the results of statistical distribution parameter fitting and ice-water classification on RADARSAT-2 data set in the Prydz Bay and Adélie Depression. Finally, the discussion and conclusion is presented in Section 5.

\section{STATISTICAL DISTRIBUTION BASSED CRF ICE- WATER DISCRIMINATION METHOD}

Statistical distributions are exploited to be integrated into the $\mathrm{CRF}$ framework to discriminate the categories of different ice type from open water using RADARSAT-2 SAR images. Statistical distribution model of SAR image aims at revealing the statistical characteristics of SAR imagery for better understanding the scattering mechanism, which is useful for
SAR image interpretation, thereby extending to other SAR application such as SAR image classification and segmentation. Benefitted from the statistical distribution in accurately modeling the SAR scattering, the statistical distribution models including Log-Normal, Weibull, Rayleigh, Gamma, and AlphaStable distributions are exploited in this section since statistical distribution has demonstrated its effectiveness in modelling the categories of different ice type and open water.

Assuming that SAR images have many uniforms and independent random scatters, according to the central limit theorem (CLT), the real and imaginary parts of the complex SAR data both follow the Gaussian distribution. Amplitude and intensity image follow Rayleigh and negative exponential distributions, respectively. In multiple-look SAR, Gamma distribution is more suitable compared to other statistical distributions. Alpha-stable distribution has been used as a successful alternative for modelling non-Gaussian data. It has also been applied to SAR images processing, e.g., for image restoration, object detection, image classification, and image fusion.

In CRF framework, let $\varphi(y)$ denote SAR span data. We have

$$
P(\varphi(y) \mid x)=\prod_{i \in S} P\left(\varphi\left(y_{i}\right) \mid x_{i}\right)
$$

Where the observations $y_{i}$ in $\varphi(y)$ is independent, and it is related to the condition $x_{i}$. Then, aiming at the incorporation with SAR statistical features for sea ice classification, the statistical distribution based CRF can be defined as

$$
\begin{aligned}
& P(x \mid y, \varphi(y))=\frac{1}{Z_{S T A-C R F}} \\
& \exp \left[\sum_{i \in S} f_{i}^{\text {unary }}\left(x_{i} \mid y\right)+\sum_{i \in S} \log P\left(\varphi(y) \mid x_{i}\right)+\sum_{i \in S} \sum_{j \in N_{i}} f_{i j}^{\text {parwise }}\left(x_{i}, x_{j} \mid y\right)\right]
\end{aligned}
$$

where $Z_{S T A-C R F}$ is the partition function for the statistical distribution based CRF model, and $y$ is the observed data, which utilized the span data in this paper. $\log P(\varphi(y) \mid x)$ can be modeled by adopting the statistical distribution model, such as Alpha-stable, Rayleigh, Weibull, to obtain the distribution parameters of different ice types. For Alpha-stable distribution, the unary potential function and the pairwise potential function adopt formula (2). $P(\varphi(y) \mid x)$ is modeled by the following Alpha-stable distribution, is expressed as

$$
P(\varphi(\omega) \mid x)=\left\{\begin{array}{l}
\exp \left\{j \mu \varphi-|\gamma \varphi|^{\alpha}[1-j \operatorname{sign}(\varphi) \beta \tan (\pi \alpha / 2)]\right\}, \alpha \neq 1 \\
\exp \left\{j \mu \varphi-|\gamma \varphi|\left[1+j \operatorname{sign}(\varphi) \beta \frac{2}{\pi} \ln (|\varphi|)\right]\right\}, \alpha=1
\end{array},\right.
$$

where sign is the sign function. $\{\alpha, \beta, \gamma, \mu\}$ are the parameters of the alpha-stable distribution. $\alpha$ is the characteristic exponent and $\beta$ is the skewed parameter. $\gamma$ is the dispersion parameter and $\mu$ stands for the location parameter. Training sample of each class represents the realization of the alpha-stable distribution. The distribution parameters can be estimated by using a pseudo-simulated annealing (PSA) (Lombardi 2015) estimator based on Markov chain Monte Carlo (MCMC) (SalasGonzalez et al., 2009) method. The alpha-stable parameters corresponding to each class are represented using formula (3). With the alpha-stable parametric distribution, the statistical features can be integrated into the CRF framework. Then, the statistical distribution based CRF model is able to capture the statistical information from the SAR images. 


\section{FRAMEWORK OF THE ICE-WATER DISCRIMINATION METHOD BASED ON STA-CRF}

We propose a supervised ice-water classification algorithm by combining statistical distribution and CRF. Samples are required for training each class to estimate the statistical distribution model parameters via logarithmic Cumulants method (MoLC) (Krylov et al., 2013). For Alpha-stable model, we use PSA Estimator since it has no analytic expression. The MoLC based parameter estimation methods of different statistical models are illustrated in Table I. The first term of Equation (2) implies that the conditional probabilities can be represented as the statistical distributions. The regularization term is introduced via $\mathrm{CRF}$, which builds a label restraint between the current pixel and its neighbourhood pixels. The classification turns to be a posterior probability maximization problem, which is equivalent to minimize the energy. To solve the energy minimization problem, we utilize a Graph Cuts (Boykov et al., 2004) optimization, as this is fast and obtains near-global optimization. The proposed statistical distribution based CRF classification algorithm is described in Figure 1.

Table I. Statistical distribution model parameter estimation.

\begin{tabular}{lll}
\hline SD model & Parameters & MoLC \\
\hline Rayleigh & $\sigma$ & $k_{1}=(\ln 2+\psi(1)) / 2+\ln \sigma$ \\
Gamma & $\mu$ & $k_{1}=\psi(L)-\ln L+\ln \mu$ \\
& & $k_{1}=\mu$ \\
Log-Normal & $\mu, \sigma$ & $k_{2}=\sigma^{2}$ \\
& & $k_{1}=\ln b-r / c$ \\
Weibull & $b, c$ & $k_{2}=\pi^{2} / 6 c^{2}$ \\
\end{tabular}

For MoLC based parameter estimation procedure, probability density function should be represented by applying Melin Transform as

$$
T(s)=M T[F](s)=\int_{0}^{\infty} x^{s-1} F(x, \theta) d x
$$

where the integral converges for $\mathrm{s}$ to calculate the $i^{\text {th }}$ derivative for obtaining the logarithmic moment estimation. The second moment of logrithmic is defined as:

$$
l_{i}=\frac{d^{i} T(s)}{d s^{i}} \mid s=1=\int_{0}^{\infty}(\ln x)^{i} T(x, \theta) d x, i=1,2, \ldots
$$

Then the logarithmic cumulants are obtained by setting $s=1$. The logarithm of equation (4) and the formula (5) are combined as:

$$
k_{i}=\frac{d^{i} \ln T(s)}{d s^{i}} \mid s=1, i=1,2, \ldots
$$

Regarding the lower moment, the logarithmic moment and logarithmic cumulants are written as:

$$
\begin{aligned}
& k_{1}=l_{1} \\
& k_{2}=l_{2}-l_{1}^{2} \\
& k_{3}=l_{3}-3 l_{2} l_{1}^{2}+2 l_{1}^{3}
\end{aligned}
$$

The logarithmic cumulants can be estimated based on the classical distribution model, which has been illustrated in Table 1. In Table I, $\psi(\cdot)$ means digamma function and $\psi(i, \cdot)$ means $i$-order polygamma function.
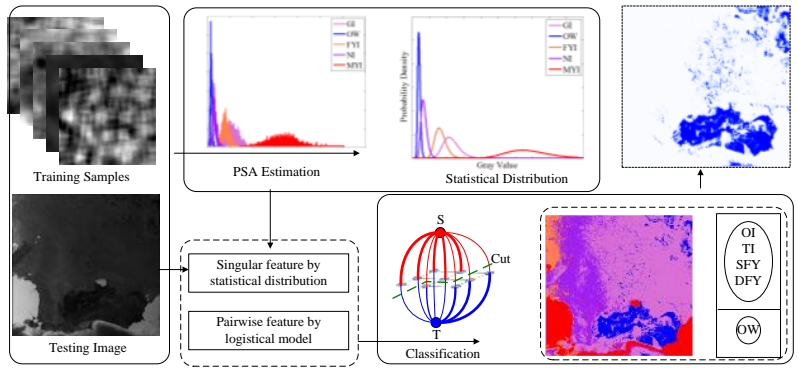

Figure 1. Framework of statistical distribution based ice-water classification algorithm.

Due to the lack of an analytical expression for the probability density function, alpha-stable distribution is usually described in the characteristic function as formula (3). Although there is no closed-form expression for the pdf of an Alpha-stable distribution, it is possible to obtain the pdf by applying a Fourier transformation to the characteristic function:

$$
S_{\alpha}(x \mid \gamma, \beta, \mu)=\int_{-\infty}^{+\infty} P(\varphi(\omega) \mid x) \exp (-j \omega x) d \omega
$$

However, it is difficult to compute Equation (4) because the characteristic function is complex and the interval of integration is infinite. Therefore, Equation (4) does not admit an analytical solution except in a few special cases. In this paper, we utilize a PSA approach to obtain the pdf of the Alpha-stable distribution. The density of alpha-stable model is given by:

$$
p(x)=S_{\alpha}(x \mid \gamma, \beta, \mu)
$$

In Bayesian scheme, we can estimate the distribution parameters via prior information and observation using the Bayesian rule

$$
p(\theta \mid X)=\frac{p(X \mid \theta) p(\theta)}{p(X)} \propto p(X \mid \theta) p(\theta)
$$

Where $p(X \mid \theta)$ denotes the posterior probability of $\theta$ given an observation $X=\left(x_{1}, \ldots, x_{j}, \ldots, x_{M}\right), p(\theta)$ is the prior probability, $p(\theta \mid X)$ is the likelihood of $X$ given $\theta$, and $p(X)$ is the prior probability of $X$. The parameter estimation procedure is considered as a missing data problem. We assume that data vector $X=\left(x_{1}, \ldots, x_{j}, \ldots, x_{M}\right)$ has been randomly drawn from $K$ subpopulations. For each variable $x_{j}$, let $z_{j}$ be an allocation variable for the unobserved or missing variable that indicates to which component $x_{j}$ belongs. Thus, $z_{j}$ will be equal to 1 if $x_{j}$ belongs to the $k^{\text {th }}$ subpopulation, or 0 otherwise. Each observation $x_{j}$ is considered to be drawn from the $k^{\text {th }}$ component described by $\theta_{k}=\left(\alpha_{k}, \beta_{k}, \gamma_{k}, \mu_{k}\right) \quad$ with probability

$$
p\left(z_{j}=k \mid x_{j}\right)=\frac{\omega_{k} S_{\alpha k}\left(x_{j} \mid \gamma_{k}, \beta_{k}, \mu_{k}\right)}{\sum_{m-1}^{K} \omega_{m} S_{\alpha m}\left(x_{j} \mid \gamma_{m}, \beta_{m}, \mu_{m}\right)}
$$

The allocation variable $z_{j}$ can be defined as follows

$$
z_{j}=\arg \max _{k} p\left(k \mid x_{j}\right)
$$

Parameters then can be updated based on $\left\{z_{j}\right\}$. The candidate parameter $\theta_{k}=\left(\alpha_{k}^{\text {new }}, \beta_{k}^{\text {new }}, \gamma_{k}^{\text {new }}, \mu_{k}^{\text {new }}\right)$ is sampled from a proposal distribution, and is accepted with probability $A_{\theta_{k}^{\text {new }}}$, where 
$A_{\theta_{k}^{\text {new }}}=\min \left(1,\left(\prod_{\substack{j=1 \\ z_{j}=k}}^{M} \frac{S_{a_{k}^{\text {new }}}\left(x_{j} \mid \gamma_{k}^{\text {new }}, \beta_{k}^{\text {new }}, \mu_{k}^{\text {new }}\right)}{\left.S_{j} \mid \gamma_{k}^{\text {old }}, \beta_{k}^{\text {old }}, \mu_{k}^{\text {old }}\right)}\right)^{\frac{1}{T(t)}} \times \frac{I G\left(\gamma_{k}^{\text {new }} \mid a_{0}, b_{0}\right) N\left(\mu_{k}^{\text {new }} \mid \xi, \kappa\right)}{I G\left(\gamma_{k}^{\text {old }} \mid a_{0}, b_{0}\right) N\left(\mu_{k}^{\text {old }} \mid \xi, \kappa\right)}\right)$

where $a_{0}$ and $b_{0}$ are parameters of the inverse gamma prior for $\gamma, \xi$ and $\kappa$ are parameters of the normal prior for $\mu$. Moreover, a normal proposal distribution $q(\cdot \mid \cdot)=N(\cdot \mid \delta, \sigma)$ is utilized in our algorithm, and its parameters are adaptively updated using estimations obtained in previous iterations: $\delta$ is set to the estimation of the previous iteration, and $\sigma$ is set to the standard deviation of the previous $L$ estimations. Adaptively updating the parameters of the proposal distribution ensures that new candidates can properly explore the entire parameter space, which further ensures that estimations rapidly converge to the true values.

For ice-water classification, the original dual-polarization RADARSAT-2 amplitude data are used to calculate the span image from $\mathrm{HH}$ and $\mathrm{HV}$ polarization, seen formula as:

$$
\text { Span }=\operatorname{sprt}\left(H H_{\text {Amplitude }}^{2}+H V_{\text {Amplitude }}^{2}\right)
$$

Then the training samples are selected from the span image with five categories including OW (Open water), TI (Thin ice), SFY (Smooth first year Ice), DFY (Deformed first year ice) and OI (Old ice). The surface colour code for each category are defined in Table II.

Training samples of each class in the span image were selected with a window size of $64 * 64$ pixels. PSA and MoLC based estimation method is exploited to obtain the distribution parameter of different statistical distribution models, which serves as the singular potential in the CRF framework and the pairwise potential are obtained by logistical model. Based on the unary and pairwise potential, the posterior probability of CRF was obtained. Then, MAP is used for predicting the label in the SAR imagery with Graph Cut method. The postprocessing procedure is carried out on the CRF result to obtain the ice-water binary classification map by integrating TI, SFY, DFY and OI into ice type.

Table II. Sea ice category color code illustration.

\begin{tabular}{c}
\hline Sea ice types \\
\hline Open water(OW) \\
Thin ice(TI) \\
Smooth first year ice(SFY) \\
Deformed first year ice(DFY) \\
Old ice (OI) \\
Land mask
\end{tabular}

\section{EXPERIMENTAL AND ANSLYSIS}

\subsection{Experimental data description}

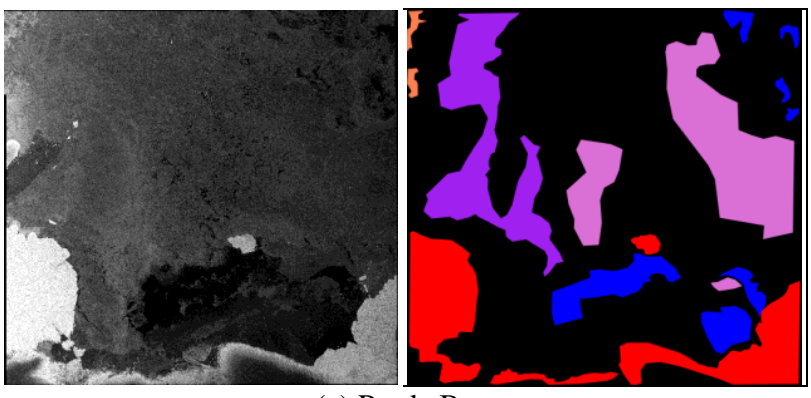

(a) Prydz Bay

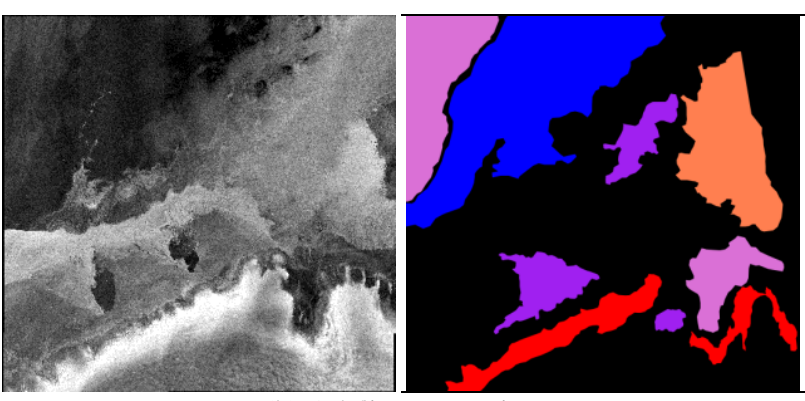

(b) Adélie Depression

Figure 2. The HH channel SAR imagery and manually selected ground-truth in Prydz Bay and Adélie Depression Table II. Experimental data description.

\begin{tabular}{|c|c|c|c|c|c|c|}
\hline \multirow[b]{2}{*}{ Data base } & \multirow[b]{2}{*}{$\begin{array}{l}\text { Acquisition } \\
\text { Time }\end{array}$} & \multicolumn{2}{|c|}{ Scene Center } & \multirow{2}{*}{\multicolumn{2}{|c|}{$\begin{array}{r}\text { Pixel } \\
\text { Polarization Spacing } \\
\text { (meter) }\end{array}$}} & \multirow[b]{2}{*}{$\begin{array}{l}\text { Image Size } \\
\quad \text { (pixel) }\end{array}$} \\
\hline & & Lon & Lat & & & \\
\hline $\mathrm{v}=\mathrm{B}$ & $3-11-$ & 5.2 & 938 & 3 HH HV & 50 & $10693 * 101$ \\
\hline $\begin{array}{l}\text { Adélie } \\
\text { epression }\end{array}$ & $014-1-6$ & & & & 50 & $10723 * 1$ \\
\hline
\end{tabular}

In this section, ice-water classification experiments are conducted on two RADARSAR-2 datasets including dual polarization SAR data with $\mathrm{HH} / \mathrm{HV}$ band in Prydz Bay area and single polarization SAR data with $\mathrm{HH}$ band in Adélie Depression area. The detailed information including image, acquisition time, location, and pixel spacing are described in Table II. For dual polarization SAR data in Prydz Bay, it is acquired on November 22, 2013 and its image size is $10693 * 10190$ pixels. For single polarization SAR data in the Adélie Depression, it is acquired on January 1, 2014. Its image size is $10723 * 10242$ pixels. The pixel spacing of the both images is 50 meters. The SAR image and corresponding ground truth images are presented in Figure 2.

For ice-water classification, statistical distributions including Alpha Stable, Weibull, Gamma, Rayleigh, Log-Normal and K based CRF methods are tested on the two RADARSAT-2 images. Besides, the SVM based classification method is also used as an experimental comparison to demonstrate the efficiency of the proposed methods. To evaluate the performance of the classification method, the ground truth image generated by manual labeling is used to calculate the overall accuracy (OA) and kappa coefficient. Quantitative assessments are provided by the accuracy reports for each type calculated in the confusion matrices shown in Table III.

\subsection{Results and analysis}

\section{1) Distribution parameter fitting}

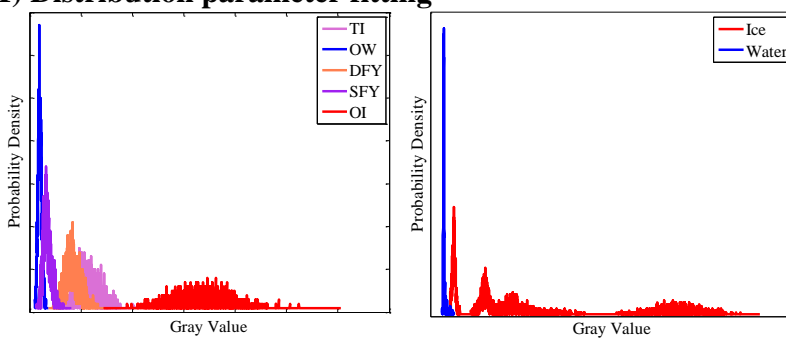

(a) Original data

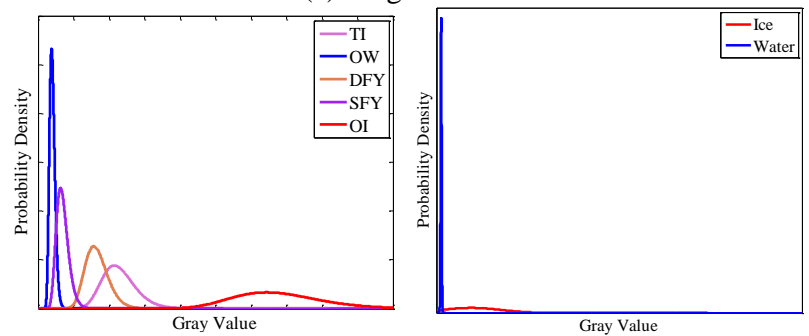


(b) Alpha-Stable

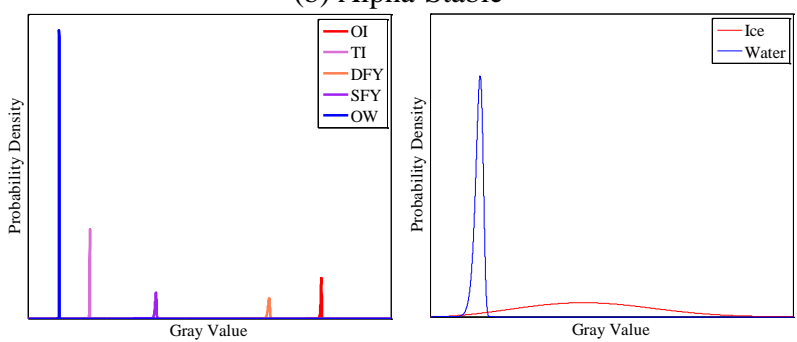

(c) Weibull

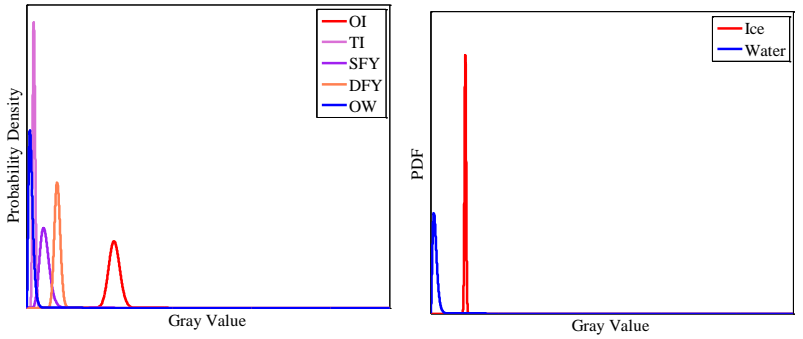

(d) Gamma

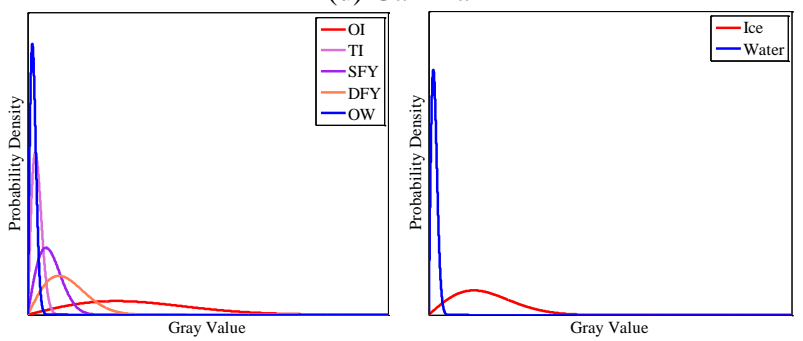

(e) Rayleigh

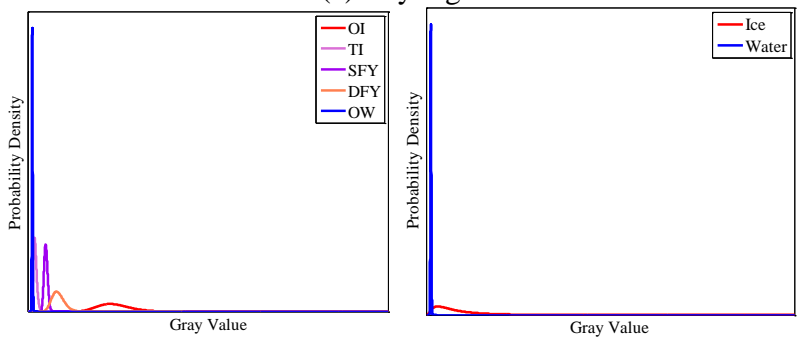

(f) Log-Normal

Figure 3. Distribution fitting result using different statistical distribution model.

For ice-water classification using statistical distribution based CRF method, the selection of distribution model shows it is of great importance for effective ice-water classification. Before the classification task we first analyze the statistical significance of different distribution respect to different ice type and open water categories. We first choose 10 sample images of each ice types and open water. Then, MoM based parameter estimation method is exploited using 50\% of the sample images to estimate the distribution parameter. Next, the parameter fit procedure is carried out on the other 50\% sample images to validate the effectiveness of different distribution model.

Distribution fitting results using different statistical distribution model are illustrated in Figure 3. The histogram in Figure 3(a) indicates that open water has a lower gray value when compared with sea ice. In the different sea ice type, OI has the highest gray value, followed by DFY and SFY. TI has the lowest gray value among the different ice types, and it has overlapped with $\mathrm{OW}$, so the critical problem in ice-water classification is to improve the accuracy of TI and OW extraction. Moreover, it is clear to see that Weibull distribution failed to model the statistical characteristics of different ice type and open water since the curves of different ice types are nearly the same. The distribution curves of OI using Gamma distribution has a sharp peak, which is different from the other distribution model, but the overlaps between OW and TI is the smallest, so it can obtain the best ice/water classification results. In Rayleigh distribution, besides OW and TI, the distribution curves between SFY and OW, DFY and OW, as well as OI and $\mathrm{OW}$ are also overlapped. We can obtain a fine result in multiple categories ice and open water classification, but it will get worse in ice/water classification using Rayleigh. Alpha-stable based method can obtain a satisfied result in both multiple categories ice and open water classification and ice/water situation.

\section{2) Sea ice classification}

Figure 4 and Figure 5 give the result of sea ice and open water classification result in Prydz and Adélie area respectively.

A. Sea ice classification in Prydz Bay area

Figure 4 gives the result of sea ice classification using different statistical distribution based CRF algorithm and feature ensemble SVM method in Prydz area. The shortcomings of SVM based method in Figure 4(a) is the misclassification of TI and OW, especially in ice edge area. For statistical distribution based method, alpha-stable based CRF method obtain the best classification result with highest precision and kappa coefficient, and it shows robust distinction of ice from open water. For Weibull distribution based CRF method, it leads to the highest misclassification rate of TI to OW with a percentage of $20.99 \%$. For Rayleigh distribution based CRF method, the misclassification rates of OI to DFY is the highest of all the methods with a percentage of $13.91 \%$. For gamma distribution based CRF method, it has the highest classification rate of SFY, but DFY have misclassified into OI. For log-normal statistical distribution based CRF method, some SYF area has been misclassified into DFY. Among the six classification method, Alpha-stable based CRF method got the highest average precision and kappa coefficient.

TABLE III. Prydz Bay sea ice classification accuracy

\begin{tabular}{|c|c|c|c|c|c|c|c|c|}
\hline Methods & Class & OW/\% & TI/\% & SFY/\% & $\overline{\mathrm{DFY} / \%}$ & OI/\% & OA/\% & Kappa \\
\hline \multirow{6}{*}{ SVM } & OW & 91.92 & 7.79 & 0.19 & 0.02 & 0.08 & \multirow{6}{*}{92.90} & \multirow{6}{*}{0.901} \\
\hline & TI & 0.67 & 96.55 & 2.75 & 0.03 & 0.00 & & \\
\hline & SFY & 0.00 & 0.01 & 99.92 & 0.06 & 0.01 & & \\
\hline & DFY & 0.00 & 0.00 & 2.14 & 92.96 & 4.90 & & \\
\hline & OI & 0.14 & 1.33 & 6.01 & 1.05 & 91.47 & & \\
\hline & OW & 90.19 & 9.69 & 0.08 & 0 & 0.04 & & \\
\hline \multirow{2}{*}{$\begin{array}{l}\text { Alpha- } \\
\text { stable }\end{array}$} & TI & 1.12 & 97.28 & 1.57 & 0.03 & 0.00 & \multirow{4}{*}{93.72} & \multirow{4}{*}{0.91} \\
\hline & SFY & 0 & 1.04 & 92.08 & 6.74 & 0.4 & & \\
\hline \multirow[t]{2}{*}{ CRF } & DFY & 0 & 1.11 & 2.94 & 93.23 & 2.71 & & \\
\hline & OI & 0.02 & 2.47 & 2.82 & 1.26 & 93.42 & & \\
\hline \multirow{5}{*}{$\begin{array}{c}\text { Gamma } \\
\text { CRF }\end{array}$} & OW & 92.45 & 1.72 & 0.05 & 0.12 & 5.66 & \multirow{5}{*}{88.03} & \multirow{5}{*}{0.861} \\
\hline & TI & 4.21 & 93.68 & 0.02 & 0.47 & 1.62 & & \\
\hline & SFY & 0.06 & 0.23 & 99.02 & 0.48 & 0.22 & & \\
\hline & DFY & 0.00 & 0.00 & 2.82 & 83.93 & 13.26 & & \\
\hline & OI & 1.07 & 1.94 & 0.45 & 7.68 & 88.86 & & \\
\hline \multirow{4}{*}{$\begin{array}{c}\text { Weibull } \\
\text { CRF }\end{array}$} & OW & 90.03 & 1.03 & 2.71 & 3.51 & 2.72 & \multirow{4}{*}{85.66} & \multirow{4}{*}{0.828} \\
\hline & TI & 20.99 & 78.45 & 0.56 & 0.00 & 0.00 & & \\
\hline & SFY & 0.00 & 2.64 & 92.13 & 4.62 & 0.61 & & \\
\hline & DFY & 0.05 & 2.62 & 0.27 & 92.03 & 5.03 & & \\
\hline
\end{tabular}




\begin{tabular}{ccccccccc}
\hline & OI & 0.11 & 0.37 & 6.21 & 1.64 & 91.67 & & \\
& OW & 93.81 & 6.14 & 0.03 & 0.03 & 0.00 & & \\
Lognormal & TI & 6.00 & 92.46 & 1.51 & 0.03 & 0.00 & & \\
CRF & SFY & 0.00 & 0.59 & 92.88 & 6.52 & 0.00 & 90.32 & 0.857 \\
& DFY & 0.00 & 1.27 & 3.38 & 89.98 & 5.37 & & \\
& OI & 0.19 & 2.28 & 2.92 & 3.05 & 91.57 & & \\
& OW & 92.07 & 3.91 & 0.25 & 0.02 & 3.74 & & \\
Rayleigh & TI & 7.51 & 91.84 & 0.63 & 0.03 & 0.00 & & \\
CRF & SFY & 0.00 & 0.00 & 97.89 & 1.50 & 0.61 & 87.54 & 0.842 \\
& DFY & 0.16 & 0.09 & 2.11 & 95.03 & 2.62 & & \\
& OI & 0.23 & 1.21 & 4.07 & 13.91 & 80.57 & & \\
\hline
\end{tabular}

The sea ice classification accuracy report in Table III indicates that the largest classification error is TI, which is misclassified as OW. The confusion matrix in table III shows that alphastable based CRF method obtains the best result in ice/water classification. The statistical distribution model in the CRF framework can describe the scattering characteristics of different ice type with different model and parameters, the effects of sea water flooding and deformation have been overwhelmed. For SVM based with GLCM contextual feature, it can provide rich features of sea ice type, while the scattering variance of different ice type is ignored.

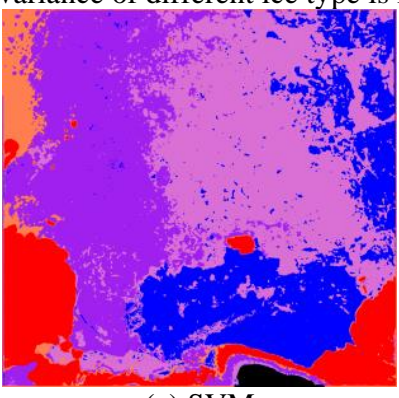

(a) SVM

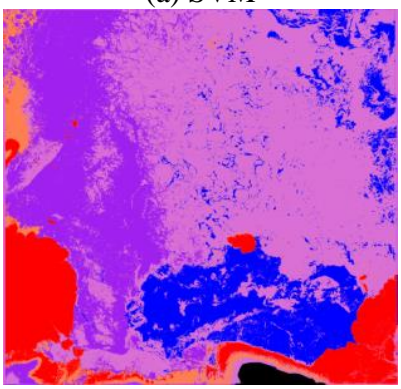

(c) Gamma CRF

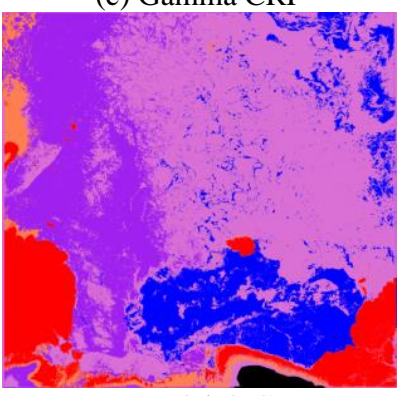

(e) Rayleigh CRF

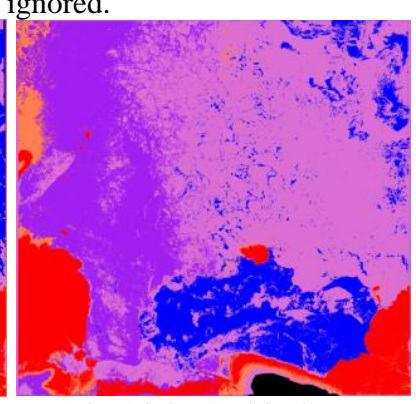

(b) Alpha-Stable CRF
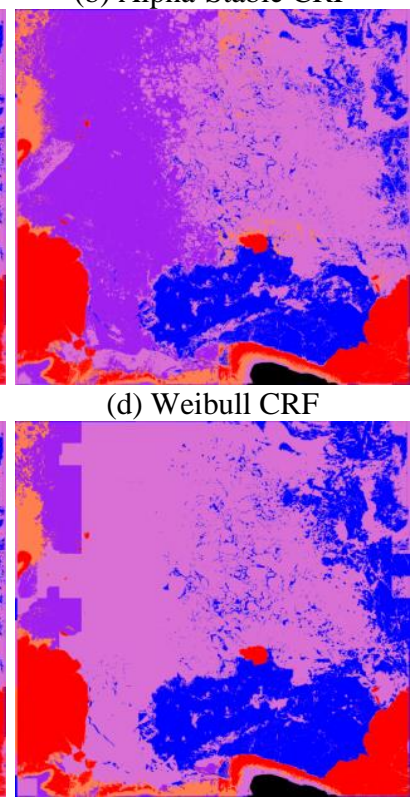

(f) Log-Normal CRF (d) Weibull CRF

Figure 4. Sea ice classification result in Prydz Bay area B. Sea ice classification in Adélie Depression area

Figure 5 shows the results of sea ice classification result in Adélie area. Figure 5(a) shows the same deficiency in poor discrimination between OW and TI as presented in Figure 4 (a) with SVM based method. The worst performance in separating
OI from DFY is gamma based CRF method with the error rate of $7.68 \%$ in table IV. SVM based method has the best result in classifying the SFY as DFY.

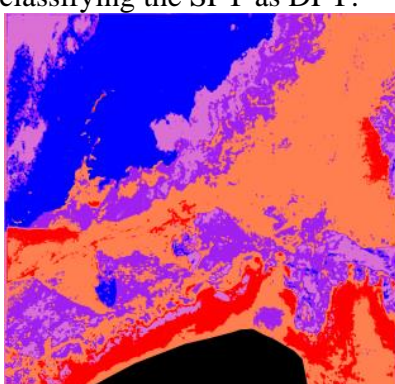

(a) SVM

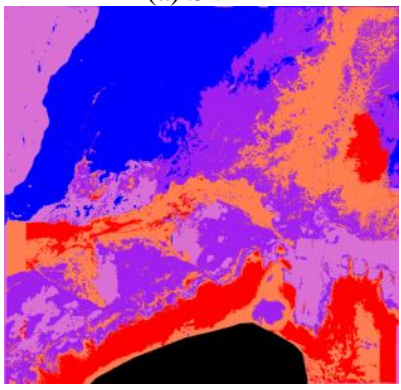

(c) Gamma CRF

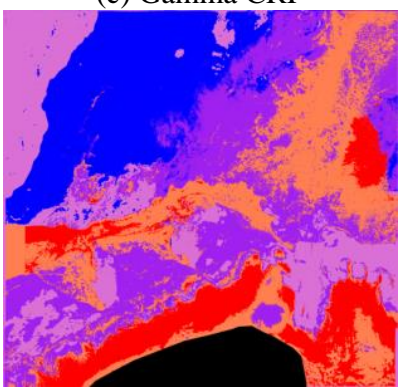

(e) Rayleigh CRF

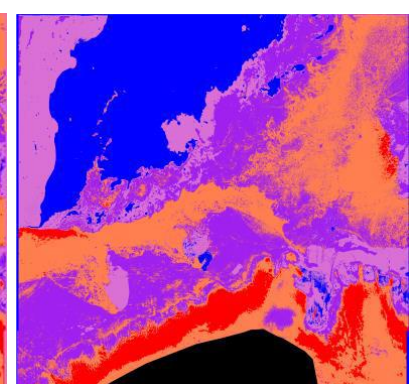

(b) Alpha-Stable CRF

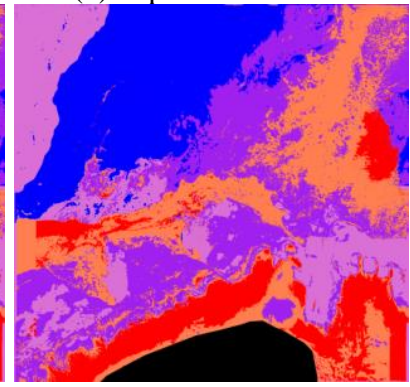

(d) Weibull CRF

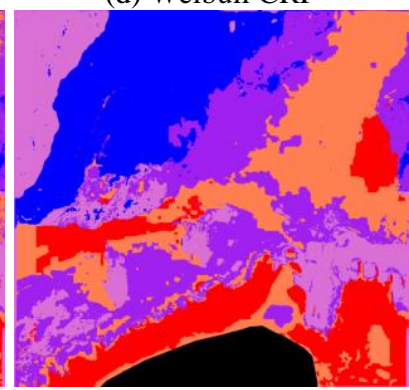

(f) Log-Normal CRF
Figure 5. Sea ice classification in Adélie Depression area

TABLE IV. Adélie Depression sea ice classification accuracy

\begin{tabular}{|c|c|c|c|c|c|c|c|c|}
\hline Methods & Class & OW/\% & $\mathrm{TI} / \%$ & SFY/\% & DFY/\% & $6 \mathrm{OI} / \%$ & $\mathrm{OA} / \%$ & Kappa \\
\hline \multirow{5}{*}{ SVM } & $\overline{\mathrm{OW}}$ & 12.38 & 65.48 & 19.58 & 2.51 & 0.05 & \multirow{5}{*}{82.11} & \multirow{5}{*}{0.760} \\
\hline & TI & 98.92 & 0.55 & 0.17 & 0.29 & 0.07 & & \\
\hline & SFY & 0.00 & 0.07 & 75.73 & 24.20 & 0.00 & & \\
\hline & DFY & 0.00 & 0.00 & 3.26 & 96.42 & 0.32 & & \\
\hline & OI & 0.00 & 13.40 & 0.00 & 0.06 & 86.54 & & \\
\hline \multirow{5}{*}{$\begin{array}{c}\text { Alpha-stable } \\
\text { CRF }\end{array}$} & OW & 95.54 & 4.33 & 0.03 & 0.06 & 0.04 & \multirow{5}{*}{89.45} & \multirow{5}{*}{0.857} \\
\hline & TI & 8.83 & 83.51 & 7.17 & 0.40 & 0.09 & & \\
\hline & SFY & 0.00 & 0.05 & 87.29 & 12.64 & 0.02 & & \\
\hline & DFY & 0.02 & 0.09 & 11.31 & 88.52 & 0.06 & & \\
\hline & OI & 0.01 & 0.00 & 0.07 & 4.59 & 95.33 & & \\
\hline \multirow{5}{*}{$\begin{array}{c}\text { Gamma } \\
\text { CRF }\end{array}$} & OW & 91.03 & 4.14 & 0.05 & 0.12 & 4.66 & \multirow{5}{*}{87.03} & \multirow{5}{*}{0.821} \\
\hline & TI & 3.21 & 87.68 & 7.02 & 0.98 & 1.11 & & \\
\hline & SFY & 0.21 & 5.02 & 85.81 & 8.74 & 0.22 & & \\
\hline & DFY & 0.00 & 0.00 & 1.20 & 88.03 & 10.77 & & \\
\hline & OI & 0.00 & 0.00 & 1.45 & 5.93 & 92.61 & & \\
\hline Weibull & OW & 92.65 & 0.04 & 3.54 & 0.03 & 3.74 & \multirow{2}{*}{86.66} & \multirow{2}{*}{0.837} \\
\hline CRF & TI & 18.32 & 81.00 & 0.66 & 0.02 & 0.00 & & \\
\hline
\end{tabular}




\begin{tabular}{ccccccccc}
\hline & SFY & 0.00 & 8.74 & 89.30 & 1.35 & 0.61 & & \\
& DFY & 0.15 & 1.62 & 1.46 & 93.87 & 2.89 & & \\
& OI & 0.43 & 0.62 & 4.64 & 3.64 & 90.67 & & \\
& OW & 92.07 & 3.91 & 0.25 & 0.02 & 3.74 & & \\
& TI & 7.51 & 81.84 & 10.63 & 0.03 & 0.00 & & \\
Lognormal & CRY & 16.00 & 0.00 & 81.89 & 1.50 & 0.61 & 80.43 & 0.742 \\
CRF & DFY & 0.16 & 10.09 & 12.11 & 75.03 & 2.62 & & \\
& OI & 0.23 & 1.21 & 4.07 & 3.91 & 90.57 & & \\
& OW & 93.80 & 6.14 & 0.03 & 0.03 & 0.00 & & \\
Rayleigh & TI & 6.00 & 85.46 & 81.51 & 0.03 & 0.00 & & \\
CRF & SFY & 0.00 & 0.59 & 86.88 & 12.52 & 0.00 & 84.48 & 0.825 \\
& DFY & 0.00 & 1.27 & 3.38 & 89.98 & 5.37 & & \\
& OI & 0.19 & 2.28 & 2.92 & 9.05 & 85.57 & & \\
\hline
\end{tabular}

Benefiting from the good performance in TI classification, the SVM based method provides the fine classification result with OA of $82.11 \%$, but the accuracy of OW classification is lonely $65.48 \%$. In terms of visual performance, the alpha-stable based CRF method retrieved the best classification results with highest accurate and kappa efficient. From Table IV, we conclude that the CRF model has confirmed the effectiveness of the CRF based methods since it can incorporate the spatial and scattering information of different ice type for classification.

\subsection{Time consuming of different algorithms}

Table V summarizes the computational time for the six sea ice classification algorithms on the two test scenes. The overall execution time of SVM based method takes the features and model training time into consideration. For the statistical distribution based CRF strategies, the calculation time is less than the SVM based method; Alpha CRF used the longest time among the statistical distribution based CRF method, followed by the Gamma CRF. However, SVM based method needs more time for the features calculation. SVM based method utilizes LIBSVM in MATLAB 2011a platform and the features are calculated by ENVI v.4.8 software. The statistical distribution based CRF are run in MATLAB 2011a platform. All testing algorithms shown in Section 4 are accomplished by a computer with an Intel Core CPU @ $2.4 \mathrm{GHz}$ and $48.00 \mathrm{~GB}$ of RAM.

Table V Computation time of different algorithms (in hours).

\begin{tabular}{ccc}
\hline Methods & Prydz Bay & Adélie Depression \\
\hline SVM based method & 3.1 & 2.7 \\
Alpha CRF & 1.3 & 1.2 \\
Gamma CRF & 1.2 & 1.1 \\
Weibull CRF & 1 & 1 \\
Rayleigh CRF & 0.9 & 0.8 \\
Log-normal CRF & 1.1 & 1 \\
\hline
\end{tabular}

\section{CONCLUSION}

In this paper, we develop different statistical distribution based CRF methods for estimating sea ice concentration. The comparisons between different statistical distributions are conducted and it can be concluded that the Alpha-stable distribution based CRF performs the best among these algorithms. Weibull distribution can get a fine discrimination of different ice type and open water, but the band width of the PDF curve is too narrow, and it fails to model the complex of the SAR image. For Rayleigh distribution, it may obtain the misclassification between ice and water, and shows the worst classification result in the five distribution based CRF model. Although Gamma distribution failed to discriminate the TI and
GI, it shows the best result in binary classification between ice and water. The Alpha-stable distribution based CRF can provide fine classification result in both multiple sea ice type and open water classification task and binary ice-water classification task. The future works will focus on the validation of sea ice concentration estimation and analysis the concentration of chlorophyll in sea ice area. Moreover, the relationship between the concentration of the chlorophyll and sea ice concentration, as well as the climate change will also be the future work.

\section{ACKNOWLEDGEMENTS (OPTIONAL)}

This study is supported by the State Key Program of National Natural Science of China, No. 41531069, the Independent research project of Wuhan University, 2017, No. 2042017kf0209, the National Natural Science Foundation of China, No. 41176173, the Polar Environment Comprehensive Investigation and Assessment Programs of China, No. CHINARE2017.

\section{REFERENCES}

Boykov, Y., \& Kolmogorov, V. (2004). An experimental comparison of min-cut/max-flow algorithms for energy minimization in vision. IEEE transactions on pattern analysis and machine intelligence, 26(9), 1124-1137.

Brucker, L., Cavalieri, D. J., Markus, T., \& Ivanoff, A. (2014). NASA Team 2 sea ice concentration algorithm retrieval uncertainty. IEEE Transactions on Geoscience and Remote Sensing, 52(11), 7336-7352.

Clausi, D. A., Qin, A. K., Chowdhury, M. S., Yu, P., \& Maillard, P. (2010). MAGIC: MAp-guided ice classification system. Canadian Journal of Remote Sensing, 36(sup1), S13S25.

Collins, M. J., \& Emery, W. J. (1988). A computational method for estimating sea ice motion in sequential Seasat synthetic aperture radar imagery by matched filtering. Journal of Geophysical Research: Oceans, 93(C8), 9241-9251.

Goodman, J. W. (1975). Statistical properties of laser speckle patterns. In Laser speckle and related phenomena (pp. 9-75). Springer Berlin Heidelberg.

Heygster, G., Alexandrov, V., Dybkjær, G., Hoyningen-Huene, V., Ardhuin, F., Katsev, I. L., ... \& Pedersen, L. T. (2012). Remote sensing of sea ice: advances during the DAMOCLES project. Cryosphere, 6(6), 1411-1434.

Krylov, V. A., Moser, G., Serpico, S. B., \& Zerubia, J. (2013). On the method of logarithmic cumulants for parametric probability density function estimation. IEEE Transactions on Image Processing, 22(10), 3791-3806.

Kuruoglu, E. E., \& Zerubia, J. (2004). Modeling SAR images with a generalization of the Rayleigh distribution. IEEE Transactions on Image Processing, 13(4), 527-533.

Li, F., Clausi, D. A., Wang, L., \& Xu, L. (2015, June). A semisupervised approach for ice-water classification using dualpolarization SAR satellite imagery. In 2015 IEEE Conference on Computer Vision and Pattern Recognition Workshops (CVPRW) (pp. 28-35). IEEE. 
Li, H. C., Hong, W., Wu, Y. R., \& Fan, P. Z. (2010). An efficient and flexible statistical model based on generalized Gamma distribution for amplitude SAR images. IEEE Transactions on Geoscience and Remote Sensing, 48(6), 27112722 .

Li, H. C., Krylov, V. A., Fan, P. Z., Zerubia, J., \& Emery, W. J. (2016). Unsupervised learning of generalized gamma mixture model with application in statistical modeling of high-resolution SAR images. IEEE Transactions on Geoscience and Remote Sensing, 54(4), 2153-2170.

Lombardi, A. M. (2015). Estimation of the parameters of ETAS models by Simulated Annealing. Scientific reports, 5 .

Moser, G., Zerubia, J., \& Serpico, S. B. (2006). SAR amplitude probability density function estimation based on a generalized Gaussian model. IEEE Transactions on Image Processing, 15(6), 1429-1442.

Oliver, C., \& Quegan, S. (2004). Understanding synthetic aperture radar images. SciTech Publishing.

Salas-Gonzalez, D., Kuruoglu, E. E., \& Ruiz, D. P. (2009). Finite mixture of $\alpha$-stable distributions. Digital Signal Processing, 19(2), 250-264.

Schervish, M. J. (2012). Theory of statistics. Springer Science \& Business Media.

Shokr, M., \& Kaleschke, L. (2012). Impact of surface conditions on thin sea ice concentration estimate from passive microwave observations. Remote Sensing of Environment, 121, 36-50.

Zhu, T., Li, F., Heygster, G., Zhang, S. (2016). Antarctic sea ice classification based on conditional random fields from RADARSAT-2 dual polarization satellite images. IEEE Journal of Selected Topics in Applied Earth Observations and Remote Sensing, 9(6), 2451-2467. 